\title{
Clinico-Pathologic Conference: Case 1
}

\author{
Márcio Ajudarte Lopes • Hyun Sil Kim • \\ Fernanda Viviane Mariano • Marcelo Brum Corrêa • \\ Neilor Teófilo de Araújo Rabelo • Pablo Agustin Vargas
}

Received: 8 September 2010/Accepted: 25 October 2010/Published online: 13 November 2010

(c) Humana 2010

\section{Clinical Presentation}

A 44 year-old, black woman was referred to the Oral Diagnosis Clinic, Piracicaba Dental School-State University of Campinas complaining of a nodule in the inferior gingiva. The patient had first noticed this nodule 4 years ago. However, she reported an increase in size and onset of pain in the last 4 months. A panoramic and occlusal radiograph was taken (Figs. 1, 2).

\section{Differential Diagnosis}

The clinical differential diagnoses for painful swelling of the right mandible in a 44 years old black female patient, could include fracture, infection, and neoplastic conditions. Although it was not mentioned, if besides these two features, loss of function had been observed, osteogenic sarcoma would be a consideration inasmuch as these tumor

M. A. Lopes $(\bowtie) \cdot$ F. V. Mariano · P. A. Vargas

Áreas de Semiologia e Patologia Oral, Departamento de Diagnóstico Oral, Faculdade de Odontologia de Piracicaba, Universidade Estadual de Campinas (UNICAMP), Av. Limeira 901, CP52, Areião, Piracicaba, São Paulo 13414-903, Brazil e-mail: malopes@fop.unicamp.br

H. S. Kim

Department of Oral Pathology, Oral Cancer Research Institute, College of Dentistry Yonsei University, Seoul, Korea

M. B. Corrêa

Department of Head and Neck Surgery, Oncology Center, Piracicaba, Brazil

N. T. d. A. Rabelo

Department of Orthopaedic Surgery, Hospital dos Fornecedores de Cana, Piracicaba, Brazil may present as a well-known triad consisting of pain, swelling and loss of function [1].

The radiological findings of the right mandibular lesion can be summarized as follow: ill-defined margin, increased bony sclerosis, patchy bone destruction, new bone formation, and periosteal reaction with a Codman's triangle. These alterations could favor the diagnosis of malignancy. Bony sclerosis was found at the periapical and cervical area of the adjacent tooth and the mandibular canal. The radioopacities seen at the periapex can be interpreted as osteomyelitis as the periapex can be the starting point of oral infection [2]. On the other hand, diffuse scattered radioopacities, which encompassed the cervical area of the tooth and the mandibular canal could not be consistent with osteomyelitis. The radio-opacity around the inferior alveolar nerve may be interpreted as sclerotic bone, which could explain the patient's pain.

Looking at the radiographs carefully, other findings can be observed. Multiple radio-opaque and radiolucent areas are present in the right maxilla and left mandible and entities to consider include a fibro-osseous lesion and Pagets disease. On the other hand, the presence of diffuse patchy bone destruction in the right body of mandible is intriguing and has to be emphasized. Although rare, osteolytic change in fibro-osseous lesion or Paget's disease must be considered. More detailed radiograph can be useful to define the disease.

Some of the alterations in the right mandible, such as bone sclerosis with bone destruction are observed in malignant conditions. Another clue for malignancy is periosteal reaction, which is seen in the ascending ramus to condylar head in the panoramic radiographic view. The periosteum is not normally visible on radiographs and the term 'periosteal reaction' refers to excess bone produced by periosteum [3]. The occlusal view demonstrates an 


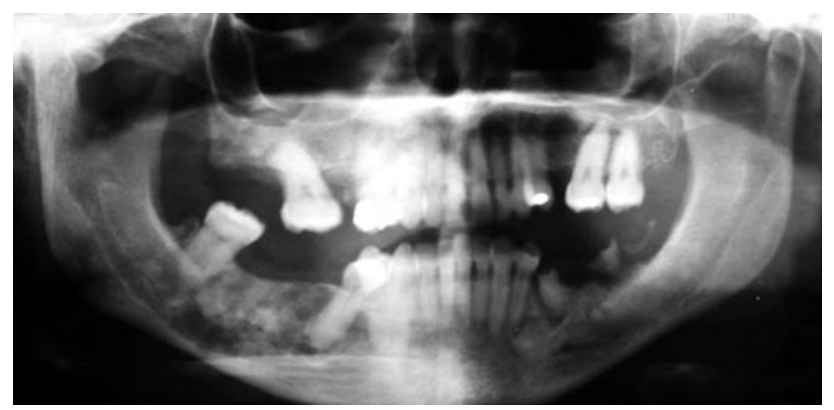

Fig. 1 Radiopaque and radiolucent area in the posterior right mandible and other multiple mixed areas in the jaws observed in the panoramic radiograph

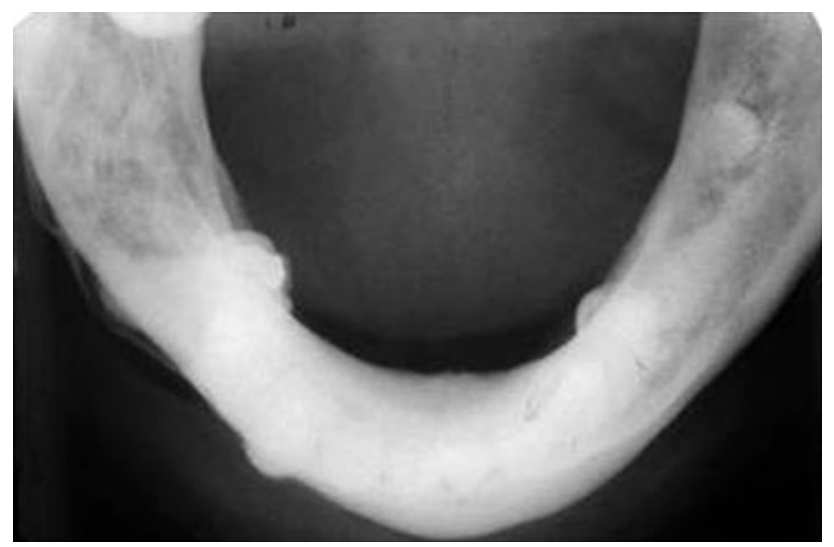

Fig. 2 Alteration in trabecular bone and extension to lingual and buccal faces in the occlusal radiograph

interrupted periosteal reaction and 'Codman's triangle'. Continuous periosteal reaction can be found in inflammatory processes or Langerhans cell histiocytosis, however, an interrupted periosteal reaction usually indicates more aggressive conditions, such as malignant tumor. The cuffshaped 'Codman's triangle' implies active form of periosteal reaction, and appears in conditions of hemorrhage or malignancy [3]. New bone formation secures the evidence supporting the malignant bone forming tumor, like osteogenic sarcoma. Therefore, the final diagnosis is malignant change in fibro-osseous lesion or Paget's disease, particularly chondrosarcoma or osteogenic sarcoma, which cannot be differentiate radiologically [4].

\section{Diagnosis and Discussion}

On extraoral examination, there was no evidence of cervical lymphadenopathy. On intraoral clinical examination a $2.0-2.5 \mathrm{~cm}$ swelling of the posterior right mandibular alveolar ridge, extending to alveolobuccal sulcus was observed (Fig. 3). Two molars had recently been extracted and the premolar was displaced. The swelling along the



Fig. 3 Intraoral view showing a swelling in the right posterior mandible

periphery was hard on palpation however, there was a fibrous consistency on the center.

The panoramic radiograph showed an extensive illdefined lytic lesion with scattered radio-opacities involving the right mandibular body approaching the inferior border. There was loss of lamina dura and widening the periodontal ligament space of the adjacent molar and premolar (Fig. 1). Additionally, there was many others mixed lesions in jaws in the dental apex. The occlusal radiograph showed an alteration in trabecular bone in the right mandibular body and a periosteal reaction or Codman's triangle of the lingual and vestibular cortical (Fig. 2).

The patient denied any systemic disease. Her family history included a brother who had died from osteosarcoma of the leg with subsequent cerebral metastases. In addition, her sister (41 years old) and her mother ( 82 years old) had several mixed lesions in the jaws with the diagnosis of the florid cemento-osseous dysplasia.

Particularly because the pain, bone expansion, and radiographic appearance of the lesion an incisional biopsy was performed. Microscopically, a hypercellular tumor composed of pleomorphic and anaplastic mesenchymal cells with atypical mitosis in a fibrocellular tissue was seen. Additionally, a dense, pink, amorphous intercellular material compatible with malignant osteoid was observed prompting the diagnosis of high-grade osteosarcoma (OS) (Fig. 4).

In order to establish the microscopic diagnosis of the other sites, curettage of a mixed area was performed during the extraction of the residual root of the first maxillary premolar, which showed a round and calcified structures in a fibrous tissue consistent with the diagnosis of cementoosseous dysplasia (Fig. 5).

The patient was referred to a head and neck surgeon, who order additional studies for staging and therapeutic planning. Biochemical analysis of serum alkaline phosphatase, calcium and phosphorus were within normal limits. A bone PET-CT (positron emission tomography-computed 


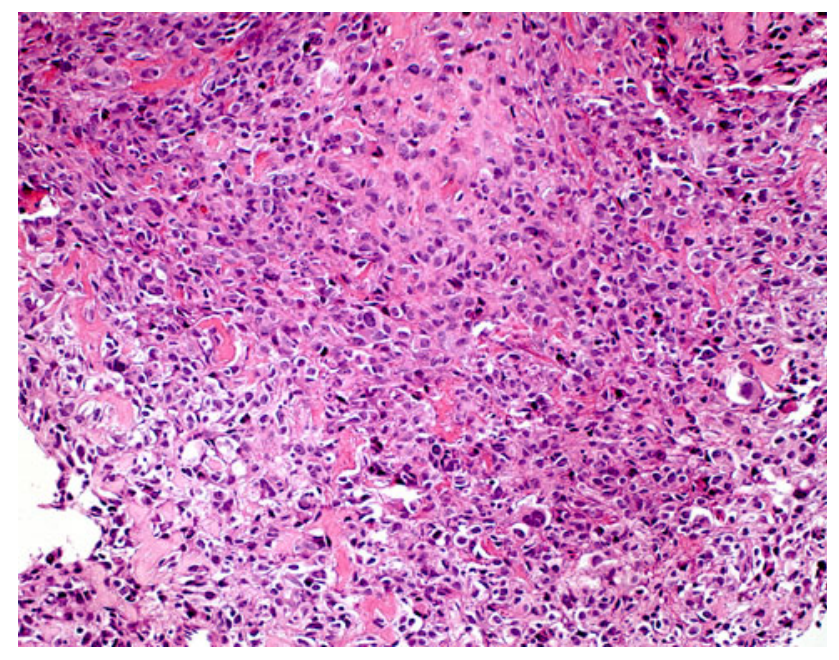

Fig. 4 Pleomorphic and anaplastic cells interspersed by osteoid $($ H.E. original magnification $\times 200)$

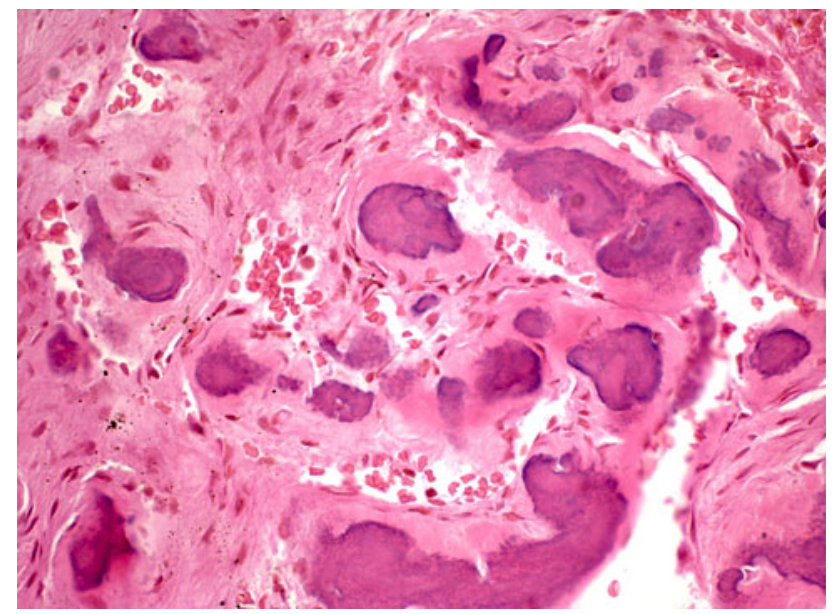

Fig. 5 Round and calcified structures in a dense connective tissue compatible with cemento-osseous dysplasia. (H.E. original magnification $\times 400$ )

tomography) or bone scintigraphy with fluoride was performed and showed uptake of radiopharmaceutical in sites of the jaws compatible with areas of cemento-osseous dysplasia, as well as in the area of the osteosarcoma (Fig. 6). However, no cervical or distant metastases were found.

The craniofacial three-dimensional tomography images showed a lesion in right mandibular body with destructive features, which caused expansion and disruption of the vestibular cortical bone (Fig. 7). Wide surgical resection (right hemimandibulectomy) was performed according to multidisciplinary planning. The mandible was microsurgically reconstructed with right fibula graft and titanium bar. A prototyping biomodel of resin was made to aid in reconstruction planning. Histopathologic examination showed surgical margins free of tumor. No adjuvant



Fig. 6 PET-CT displaying high uptake in the right mandibular body and in other several sites of the jaw bones

therapy, such as chemotherapy or radiotherapy was recommended. After 14 months of follow-up, no recurrence or metastasis has been detected (Fig. 8).

Osteosarcoma is a primary malignant bone tumor characterized by the formation of osteoid by tumor cells. It affects more frequently long bones of young people. The occurrence in the head and neck region is rare accounting for about $10 \%$ of all OS. OS is more common in males, presenting usually in the 3rd or 4th decades of life and mainly affects the mandible. The most common clinical features are swelling and pain as observed in the current case, however, change in teeth position was also observed. Radiographic appearance may be purely osteolytic, osteogenic, mixed, or with sunray pattern. When OS affects the jaws a widening of periodontal ligament space and of the inferior mandibular canal, and loss of lamina dura can be observed as found in the present case [5].

OS can arise de novo or in benign precursors lesions, which may be: genetic predisposing diseases, sporadic premalignant tumors (Paget's disease, giant cell tumor, 


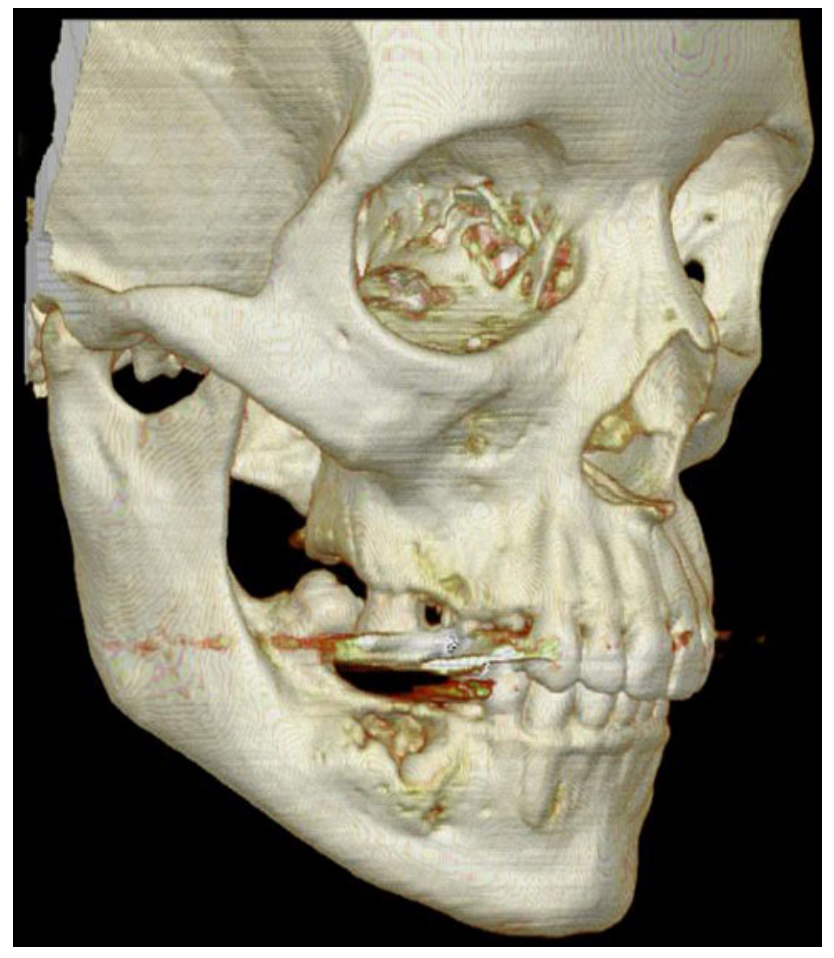

Fig. 7 Expansion and destructive features of the cortical bone observed in the craniofacial three-dimensional tomography

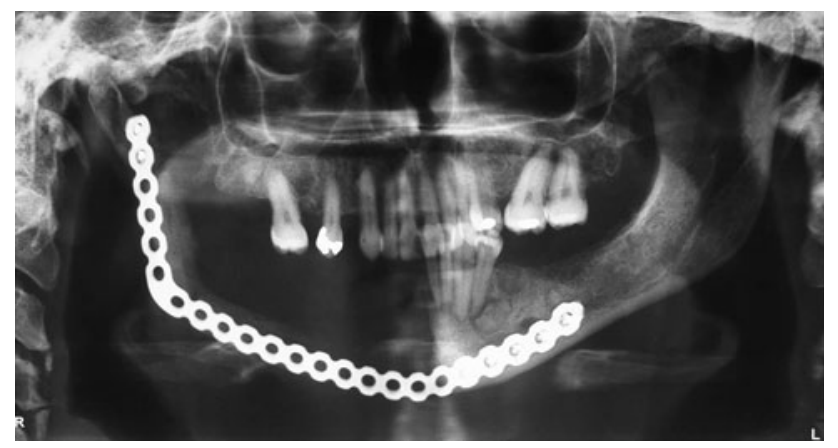

Fig. 8 Panoramic radiograph showing post-operative aspects with bone graft and titanium bar

chronic osteomyelitis, osteoblastoma, chondroid neoplasms, osteochondroma, synovial chondromatosis, enchondroma, and fibrous dysplasia), and sarcoma post-radiation [6]. Among the related lesions, fibrous dysplasia (FD) and Paget's disease are bone disease considered as important predisposing factors for developing osteosarcoma.

Fibrous dysplasia is a benign fibro-osseous lesion divided into two major types: monostotic and polyostotic. Malignant change in FD is extremely rare with a reported frequency of about $0.5 \%$ for monostotic FD and $4 \%$ for McCune-Albright syndrome [7]. The most frequent sites involved in FD malignant changes were craniofacial bones, proximal femur, humerus, pelvis, and tibia. FD is usually asymptomatic, and therefore, when there is pain without prior trauma, malignant transformation should be considered. Radiographically, osteolytic lesions with poorly delineated borders within or near the area with a groundglass appearance and cortical destruction with soft tissue extension might indicate a malignant change in FD [7].

Paget's disease of bone is a progressive skeletal disorder of unknown etiology characterized by rapid resorption and deposition of bone with resulting in deformity and impairment of the affected bone [8]. The polyostotic disease usually affects the pelvis, spine, femur, and skull, while the monostotic disease may occur in the tibia, ilium, femur, skull and less frequently the mandible. The age of the affected patients is 40 years old, and there is predominance in white and male individuals. The serum alkaline phosphatase and serum calcium is increased or normal in patients with Paget's disease [8].

Cheng et al. [8] reported a case of an osteosarcoma arising in Paget's disease with areas associated with florid cemento-osseous dysplasia (FCOD). FCOD has many similar clinical, radiographic and scintigraphic findings of Paget's disease. However, FCOD is localized only in the jaw bones and has different race and gender predilection. Studies are necessary to investigate if patients with FCOD have similar genetic abnormalities seen in Paget's disease of bone and consequently have potential for malignant transformation.

Schneider et al. [9] reported a malignant spindle cell tumor arising in the mandible of a 54 year-old black woman with florid cemento-osseous dysplasia. The case was the first reported example of a sarcoma arising in association with FCOD. Melrose and Handlers [10], described in a brief abstract a 36 year-old black woman with florid cemento-osseous dysplasia that developed osteosarcoma. The history and clinical features were very similar with current case.

The cause of FCOD is unknown, affects typically middle-age women African and Asian descent, and the hereditary nature was demonstrated in few studies. In the current case the patient reported that her sister and her mother developed FCOD. The lesions of FCOD are multiple, the borders are round to lobulated in shape and vary from well-defined to poorly defined. It is common to see a radiolucent rim surrounding the lesions, and the alterations are confined to the toothbearing regions. In the present case the lesions are lytic with radiopaque points, well defined borders and localized close to dental apex.

The association of OS and FCOD is extremely rare and could simply represent a collision between a benign and malignant lesion or may represent OS arising in FCOD. However, as the doubt persist it is important that patients with FCOD be followed and monitored in a regular basis. 


\section{References}

1. August M, Magennis P, Dewitt D. Osteogenic sarcoma of the jaws: factors influencing prognosis. Int J Oral Maxillofac Surg. 1997;26:198-204.

2. Suei Y, Tanimoto K, Taguchi A, et al. Chronic recurrent multifocal osteomyelitis involving the mandible. Oral Surg Oral Med Oral Pathol. 1994;78:156-62.

3. Armstrong P, Wastie ML. London: diagnostic imaging. London: Blackwell Scientific Publications; 1992. p. 297-329.

4. Huvos AG. Clinicopathologic spectrum of osteogenic sarcoma. Recent observations. Pathol Annu. 1979;14:123-44.

5. Nissanka EH, Amaratunge EAPD, Tilakaratne WM. Clinicopathological analysis of osteosarcoma of jaw bones. Oral Dis. 2007;13:82-7.
6. Horvai A, Unni KK. Premalignant conditions of bone. J Orthop Sci. 2006;11:412-23.

7. Hoshi M, Matsumoto S, Manabe J, et al. Malignant change secondary to fibrous dysplasia. Int J Clin Oncol. 2006;11:229-35.

8. Cheng YSL, Wright JM, Walstad WR, et al. Osteosarcoma arising in Paget's disease of the mandible. Oral Oncol. 2002;38: 785-92.

9. Schneider LC, Dolinsky HB, Grodjesk JE, et al. Malignant spindle cell tumor arising in the mandible of a patient with florid osseous dysplasia. Oral Surg Oral Med Oral Pathol Oral Radiol Endod. 1999;88:69-73.

10. Melrose R, Handlers J. Osteosarcoma ex florid osseous dysplasia: report of a case. Oral Surg Oral Med Oral Pathol. 2003;96:296. 